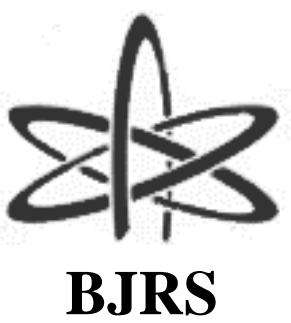

\author{
BRAZILIAN JOURNAL \\ $\mathrm{OF}$ \\ RADIATION SCIENCES \\ 08-01 (2020) 01-15
}

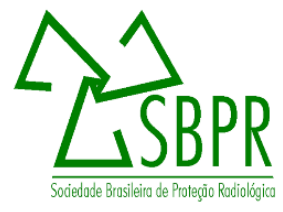

\title{
Caracterização dosimétrica do LiF-NRPB para calibração de aplicadores clínicos de betaterapia
}

\author{
Litvac D., Caldas L. V. E. \\ Instituto de Pesquisas Energéticas e Nucleares / Comissão Nacional de Energia Nuclear (IPEN/CNEN-SP), \\ Av. Prof. Lineu Prestes 2242, 05508-000, São Paulo, SP, Brasil \\ daniel.litvac@ipen.br
}

\section{RESUMO}

A National Radiological Protection Board era uma instituição que trabalhava com radioproteção pessoal e utilizava materiais dosimétricos de LiF. Um destes materiais, empregado antigamente na rotina de trabalho da instituição, consiste de uma placa de liga de alumínio, onde dois discos de politetrafluoroetileno carregados com fluoreto de lítio são retidos por anéis de alumínio. São oficializados, no Sistema Único de Saúde, procedimentos radioterápicos em betaterapia dérmica, betaterapia oftalmológica e betaterapia para profilaxia de pterígio. Na norma NN 3.01, "Diretrizes Básicas de Proteção Radiológica", exige-se a calibração regular destes aplicadores. $O$ objetivo deste trabalho foi caracterizar dosimetricamente as amostras de LiF-NRPB, verificar se são adequadas para utilização em um sistema para calibrações de aplicadores clínicos de betaterapia e identificar se há a possibilidade de novas aplicações dosimétricas. 


\begin{abstract}
The National Radiological Protection Board was an institution working with personal radiation protection using LiF as dosimetric materials. One of these materials, formerly employed in the institution's working routine, consists of an aluminum alloy plate, where two lithium fluoride with polytetrafluoroethylene discs are retained by aluminum rings. Radiotherapeutic procedures in dermal beta therapy, ophthalmological beta therapy and pterygium prophylaxis are formalized in the Unified Health System. "Basic Radiological Protection Guidelines" require regular calibration of these applicators. The objective of this work was to characterize dosimetrically the LiF-NRPB samples, to verify if they are suitable for use in a system for the calibration of clinical beta therapy applicators and to identify if there is the possibility of new dosimetric applications.
\end{abstract}

Keywords: Lithium Fluoride, Beta therapy, Thermoluminescense. 


\section{INTRODUÇÃO}

A instituição britância National Radiological Protection Board (NRPB) foi uma empresa que trabalhou em torno dos anos 80 com radioproteção e monitoração pessoal; hoje a instituição é conhecida como Health Protection Agency (HPA) e faz parte da Public Health England (PHE) e ainda trabalha com radioproteção. Ela utilizava materiais dosimétricos na prestação de serviços clínicos, industriais e monitoração individual. Um destes materiais foi o Fluoreto de Lítio misturado ao politetrafluoretileno (LiF-NRPB) [1].

Estas amostras de LiF-NRPB são semelhantes a amostras mencionadas na literatura. Dentre as mais comuns e com comportamento TL semelhante, $\mathrm{LiF}$ : $\mathrm{Mg}$, Ti misturado ao PTFE e LiF: $\mathrm{Mg}, \mathrm{Cu}, \mathrm{P}+$ PTFE são duas das que mais aparecem em artigos científicos que vinculam a utilização deste tipo de amostra dosimétrica à área médica [2, 3]. Gonzalez e Azorín [4] produziram LiF: $\mathrm{Mg}, \mathrm{Cu}, \mathrm{P}+\mathrm{PTFE}$ e realizaram um estudo da resposta TL deste material para fontes radioativas beta do tipo ${ }^{90} \mathrm{Sr} /{ }^{90} \mathrm{Y}$ e Azorín et al. [5] desenvolveram pastilhas de LiF misturado ao PTFE para monitoração pessoal. Outros artigos aplicaram este tipo de material à dosimetria na área de radioterapia [6] e à dosimetria na área de radiodiagnóstico [7]. Apesar de serem semelhantes ao LiF-NRPB, existe uma particularidade na curva da resposta termoluminescente apresentada neste trabalho que os outros materiais não apresentam [1]. Para a avaliação dosimétrica do LiF-NRPB, a betaterapia foi escolhida como tema de caracterização.

A betaterapia é um tipo de braquiterapia que o Sistema Único de Saúde e serviços particulares implantaram para suprir a demanda para tratamento de queloides, pterígios e profilaxias. Aplicadores clínicos de ${ }^{90} \mathrm{Sr} /{ }^{90} \mathrm{Y}$, com meia-vida de 28,8 anos [8], são ainda utilizados para tratamentos dermatológicos e oftálmicos no Brasil. A calibração e a recalibração periódica destes aplicadores, para verificação da taxa de dose absorvida, são essenciais para garantir segurança nos tratamentos clínicos [9].

O objetivo deste trabalho foi avaliar a resposta termoluminescente de amostras de LiF-NRPB por meio de um sistema para calibrações de aplicadores clínicos de betaterapia e avaliar as condições de uso destas amostras para outras aplicações. 


\section{METODOLOGIA}

\subsection{Amostras dosimétricas}

Foram utilizadas pastilhas de LiF-NRPB que consistem de uma placa de liga de alumínio, onde dois discos de politetrafluoroetileno carregados com fluoreto de lítio são retidos por anéis de alumínio [1]. Estes materiais foram recebidos no IPEN por doação do NRPB e estavam disponíveis no Centro de Metrologia das Radiações, empacotados e conservados, apresentando ótimas condições para uso. Há dois tipos de pastilhas dentro deste grupo de amostras de LiF, em termos de massa por unidade de área, um de $40 \mathrm{mg} / \mathrm{cm}^{2}$ com espessura de $0,1 \mathrm{~mm}$ e o outro de $90 \mathrm{mg} / \mathrm{cm}^{2}$ com espessura de 0,7 mm [1]. Cada amostra de LiF-NRPB estava presa a placas de metal e envoltas pelos anéis de alumínio (Figura 1); foram retirados por meio de um alicate e cortados em 40 pequenas pastilhas de $4 \mathrm{~mm}$ de diâmetro com um furador para couro.

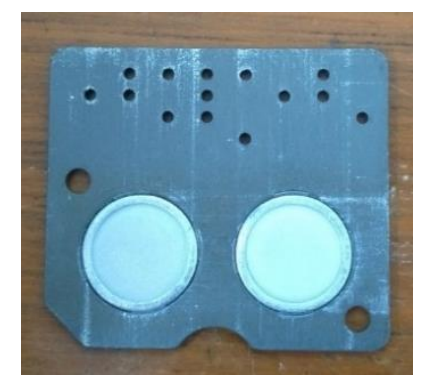

Figura 1 : Placa de liga de alumínio com dois discos de politetrafluoroetileno carregados com fluoreto de lítio.

Foi realizada a medição da massa das 40 pastilhas por meio da balança Mettler Toledo modelo AB204-S, para a possível necessidade de correção dos dados obtidos e para a determinação da sensibilidade TL do material.

Para a irradiação do material e posterior seleção das amostras, foi utilizado um suporte de acrílico de $10 \times 10 \times 2 \mathrm{~cm}^{3}$ de espessura, com rebaixos circulares para posicionar as pastilhas. Para o tratamento térmico, foi utilizado um suporte de cobre, no formato de bandeja cúbica, com dimensões de $10 \times 6 \mathrm{~cm}^{2}$. 


\subsection{Caracterização dosimétrica}

Para todas as medições foram realizados vários ciclos de medição, tratamento térmico e os seguintes testes, recomendados por McKeever [2] e Furetta [3]:

- Reprodutibilidade de resposta;

- Linearidade da resposta das amostras para diferentes doses absorvidas;

- Homogeneidade da resposta;

- Dependência angular da resposta das amostras para corrigir discrepâncias entre medições de dosímetros com diferentes inclinações angulares;

- Para a realização de cada medição TL, esperou-se 1 h após cada irradiação.

\subsection{Sistema leitor}

Foi usado o sistema leitor Risö, modelo TL/OSL-DA-200, com taxa de aquecimento de $0,1^{\circ} \mathrm{C} / \mathrm{s}$ a $10^{\circ} \mathrm{C} / \mathrm{s}$, para a avaliação TL das pastilhas de LiF-NRPB.

\subsection{Sistema de radiação}

Foram utilizados três sistemas de radiação beta para caracterização do material dosimétrico. $\mathrm{O}$ primeiro $\left({ }^{90} \mathrm{Sr} /{ }^{90} \mathrm{Y}\right)$ faz parte do sistema leitor Risö, modelo TL/OSL-DA-200, com taxa de dose absorvida de $83 \mathrm{mGy} / \mathrm{s}$ (2019). O segundo é uma fonte do sistema padrão secundário BSS2 de ${ }^{90} \mathrm{Sr} /{ }^{90} \mathrm{Y}$ com taxa de dose de $119 \mu \mathrm{Gy} \mathrm{s}{ }^{-1}$ (2019), sendo o certificado do fabricante de 27/04/2005, Amersham Buchler, à distância fonte-detector de $11 \mathrm{~cm}$, com taxa de dose no ar de 175,9 $\mu \mathrm{Gy} / \mathrm{s}$ e taxa de dose na pele $(\mathrm{d}=0)$ de $192,5 \mu \mathrm{Gy} / \mathrm{s}$. O terceiro é o sistema padrão secundário BSS1 $\left({ }^{90} \mathrm{Sr} /{ }^{90} \mathrm{Y}\right)$, com taxa de dose no ar de 207,3 $\mu \mathrm{Gy} / \mathrm{s}$, à distância de $11 \mathrm{~cm}$ (2019), sendo o certificado do fabricante de 04/02/1981, à distância fonte-detector de $11 \mathrm{~cm}$, com taxa de dose no ar de $518,4 \mu \mathrm{Gy} / \mathrm{s}$. 


\subsection{Tratamento térmico}

Um forno do tipo mufla por micro-ondas Provecto Analítica, modelo MFLO1000, foi utilizado com uma taxa de aquecimento de $47^{\circ} \mathrm{C} / \mathrm{min}$ e incerteza de $\pm 3^{\circ} \mathrm{C}$. As amostras foram tratadas, inicialmente, a $300{ }^{\circ} \mathrm{C}$ durante $1 \mathrm{~h}$, seguindo uma parte da metodologia de Dennis et al. [1].

\subsection{Calibração de um aplicador clínico}

Foi calibrado um aplicador clínico dermatológico Amersham, modelo SIQ18, com dimensões $2 \mathrm{~cm}$ x $2 \mathrm{~cm}$, e com 0,027Gy/s de taxa de dose na superfície da pele, considerando o decaimento para o ano de 2019 (certificado de calibração de 08/11/1968) .

Os principais documentos envolvendo fontes de braquiterapia afirmam que a calibração dos aplicadores deve ser de acordo com a taxa de dose depositada no tecido ou na água. A margem de incerteza ou coeficiente de variação (C.V.) deve ser de no máximo $5 \% \quad(k=1)$ dentro da reprodutibilidade de resposta e devem ser consideradas as incertezas do tipo A e B para a incerteza expandida $(\mathrm{k}=2)$. Os dosímetros devem ser calibrados de acordo com um material radioativo padrão primário ou secundário e a distância referência fonte-detector deve ser de $1 \mathrm{~mm}$ com o dosímetro centralizado no eixo principal do aplicador. A norma ISO [9] também recomenda que o detector possua no máximo $1 \mathrm{~mm}$ nas dimensões, mas neste estudo foram utilizadas amostras de LiF-NRPB com 4 mm de diâmetro. A Figura 2 apresenta o ajuste experimental utilizado no trabalho.

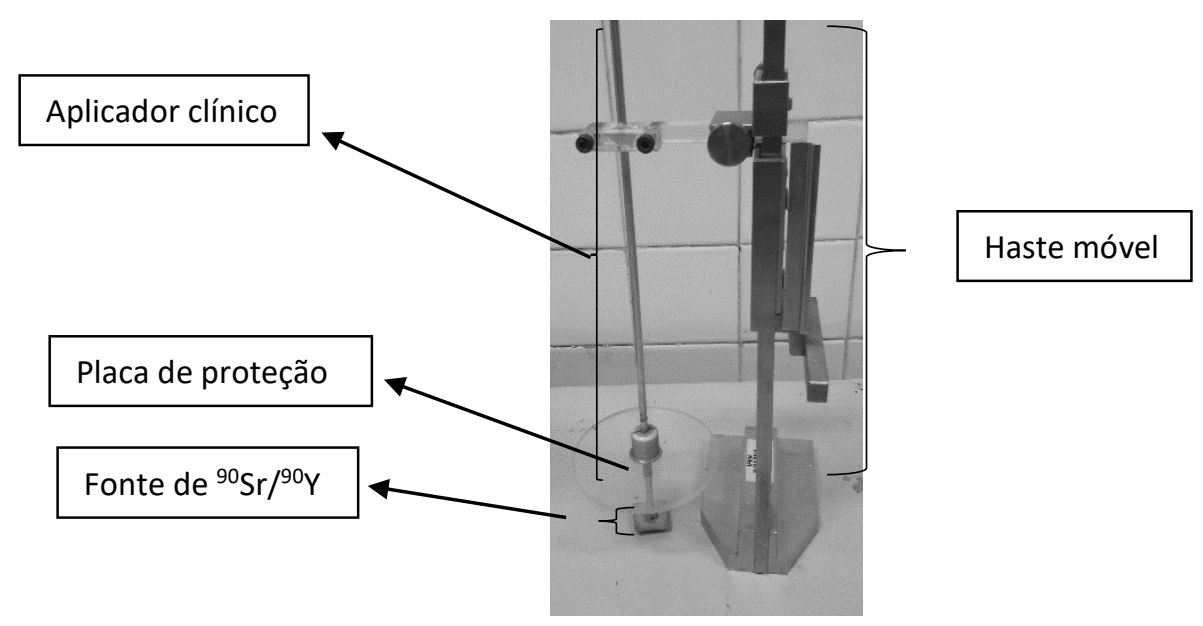

Figura 2: Ajuste do aplicador clínico por meio de haste móvel, posicionado a $1 \mathrm{~mm}$ da amostra. 


\subsection{Sensibilidade TL}

Foi determinada a sensibilidade das amostras utilizando-se a Equação 1, onde $\mathrm{M}_{\mathrm{TL}}$ é a medição TL, D a dose absorvida, m a massa da amostra e f' é a sensibilidade da amostra [3]:

$$
f^{\prime}=\left(M_{T L} / D \cdot m\right)
$$

\subsection{Incertezas para calibração de aplicadores clínicos}

Neste trabalho manteve-se um nível de confiança de 95\%. A Equação 2 apresenta a incerteza combinada, onde as incertezas do tipo A e tipo B são consideradas para a obtenção de uma incerteza total relacionada à medição [9].

$$
\sigma_{\text {combinada }}^{2}=\sigma_{\text {A }}^{2}+\sigma_{\text {B }}^{2}
$$

onde $\sigma_{\mathrm{A}}$ são as incertezas do tipo $\mathrm{A}$ e $\sigma_{\mathrm{B}}$ são as incertezas do tipo $\mathrm{B}$, relacionadas a seguir:

- Incerteza da balança: $\pm 0,1 \%$;

- Incerteza da leitora RISÖ TL/OSL-DA20 para medições TL: \pm 0,05\%;

- Incerteza na temperatura do tratamento térmico: $\pm 3 \%$;

- Incerteza dos sistemas de radiação BSS1 e BSS2: $\pm 2 \%(\mathrm{k}=2)$;

- Incerteza do cronômetro digital: $\pm 1 \%$.

Para avaliar as melhores respostas TL quanto ao teste reprodutibilidade, foram determinados, para cada amostra, os valores dos C.V. [3], que é a razão entre a incerteza do tipo A e o valor médio das medições TL realizadas no teste.

Além da incerteza combinada apresentada na Equação 2, foi considerada a incerteza expandida $(\mathrm{k}=2)$ do sistema de radiação BSS2 para a calibração dos aplicadores clínicos de betaterapia.

\section{RESULTADOS E DISCUSSÃO}

De acordo com Dennis et al. [1], as amostras devem passar por um tratamento térmico de $80{ }^{\circ} \mathrm{C} / 16 \mathrm{~h}$ e, em casos de altas doses, também deve ser previamente realizado um tratamento de $300{ }^{\circ} \mathrm{C} / 1 \mathrm{~h}$. Como não há a possibilidade de se realizar o tratamento durante $16 \mathrm{~h}$ no IPEN por 
motivos técnicos, a caracterização destas pastilhas foi efetuada com uma adaptação, por meio de testes, com o tratamento de $300^{\circ} \mathrm{C} / 1 \mathrm{~h}$ e $100^{\circ} \mathrm{C} / 2 \mathrm{~h}$ que é um tratamento utilizado comumente em amostras de LiF [3].

Diferentemente do que foi reportado por Dennis et al. [1], algumas das curvas TL apresentam o pico principal evidente apenas após o tratamento a $300^{\circ} \mathrm{C} / 1 \mathrm{~h}$. As amostras que foram tratadas apenas a $300^{\circ} \mathrm{C}$, e não apresentaram pico principal, possuem a forma mostrada na Figura 3.

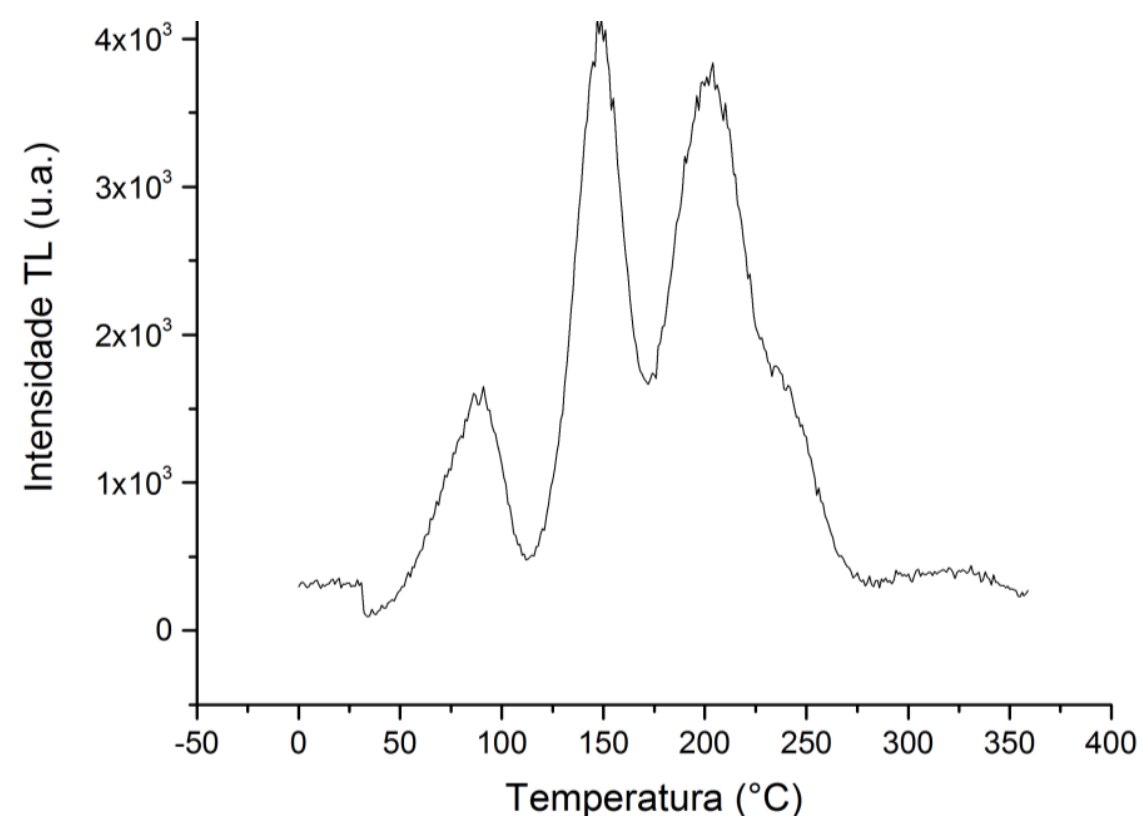

Figura 3: Curva TL de uma amostra de LiF-NRPB sem a presença evidente do pico principal, irradiada com 1 Gy, no sistema RISÖ TL/OSL-DA20 de radiação beta $\left({ }^{90} \mathrm{Sr} /{ }^{90} \mathrm{Y}\right)$. Taxa de aquecimento: $10^{\circ} \mathrm{C} / \mathrm{s}$. Sistema leitor TL: RISÖ TL/OSL-DA2O.

Este tipo de curva não é reprodutível porque as armadilhas eletrônicas de baixas temperaturas do material estão instáveis com a falta do tratamento térmico [3]; assim, não é aplicável em dosimetria.

A curva da Figura 4 apresenta o pico principal em torno de $250{ }^{\circ} \mathrm{C}$ e é a curva que representa a resposta TL da mesma amostra da Figura 3 após ter sido submetida ao tratamento térmico completo. Esta curva é muito semelhante à curva obtida por Azorín et al. [5]. 


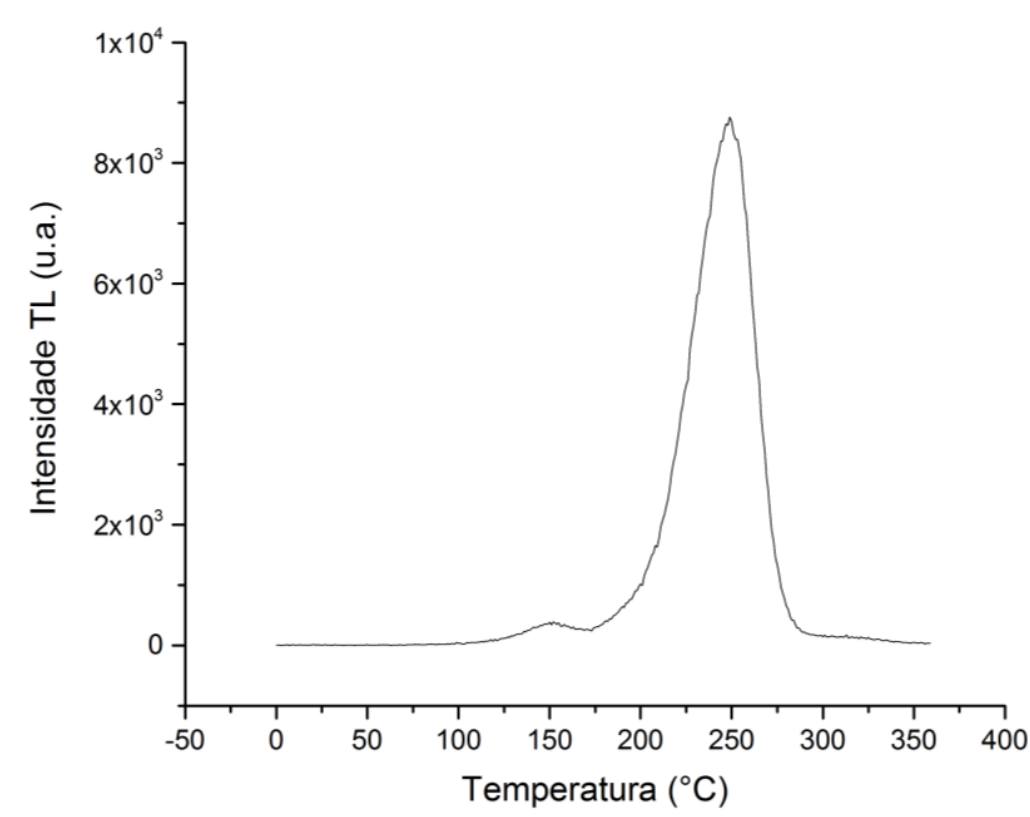

Figura 4: Curva TL de uma amostra de LiF-NRPB com o pico principal evidente, irradiada com 1 Gy, no sistema RISÖ TL/OSL-DA20 de radiação beta $\left({ }^{90} \mathrm{Sr} /{ }^{0} \mathrm{Y}\right)$. Taxa de aquecimento: $10^{\circ} \mathrm{C} / \mathrm{s}$. Sistema leitor TL: RISÖ TL/OSL-DA20.

\subsection{Reprodutibilidade e homogeneidade de resposta}

As 40 pastilhas de LiF-NRPB foram avaliadas quanto à reprodutibilidade de resposta. As medições e as incertezas relacionadas para 6 ciclos de medição das amostras foram realizadas por meio do sistema RISÖ TL/OSL-DA20 de radiação beta $\left({ }^{90} \mathrm{Sr} /{ }^{90} \mathrm{Y}\right)$, com taxa de aquecimento de $10^{\circ} \mathrm{C} / \mathrm{s}$ e no sistema leitor TL, RISÖ TL/OSL-DA20 (1 Gy).

Por meio da Equação 1, foi construído o histograma (Figura 5) correspondente à sensibilidade das 40 amostras. 


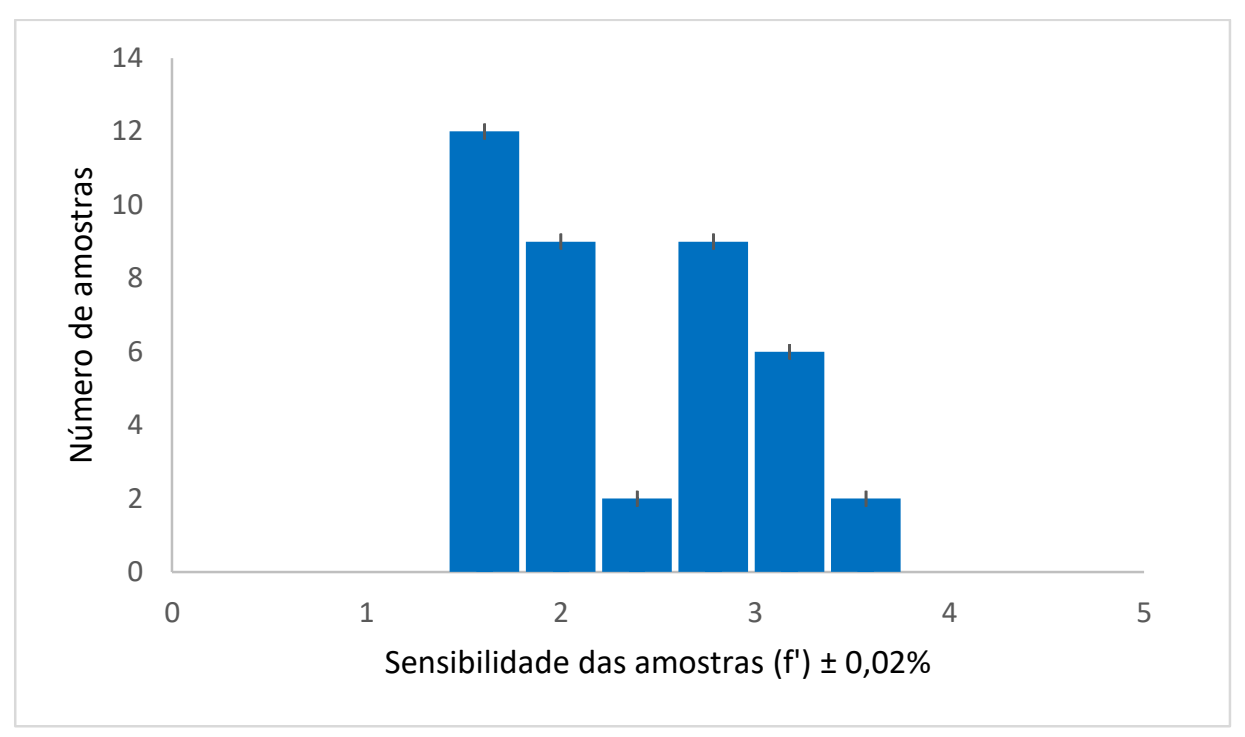

Figura 5: Histograma da variação da sensibilidade da resposta TL das 40 amostras de LiF-NRPB relacionados ao teste de reprodutibilidade.

Quatro amostras de melhores características dosimétricas para os testes de reprodutibilidade de resposta e homogeneidade de resposta foram selecionadas.

Os valores de C.V. relacionados às amostras 1 a 4 são, respectivamente, $0,75 \%, 1,25 \%, 0,73 \%$ e $1,01 \%$. Estes valores estão abaixo de 5\%, valor recomendado pela norma ISO [9].

Para o teste de homogeneidade da resposta TL das 4 amostras dosimétricas de LiF-NRPB, o valor médio de intensidade TL foi de 14,42 u.a. e o conjunto de variação da resposta percentual foi de 5,4\%, valor relacionado à diferença percentual entre o maior valor de resposta TL de um grupo de amostras e o menor valor de resposta TL do grupo [3].

\subsection{Curva dose-resposta}

Para as amostras de LiF-NRPB, foi determinada a curva dose-resposta TL das 4 amostras selecionadas (Figura 6). A curva apresentou ajuste linear e seu coeficiente de correlação linear foi de 0,9976. A linearidade de resposta foi avaliada em um intervalo de dose de 0,5 Gy a 3 Gy, considerando que os tratamentos utilizados em betaterapia podem variar de 2,0 a 3,0 Gy [9]. 


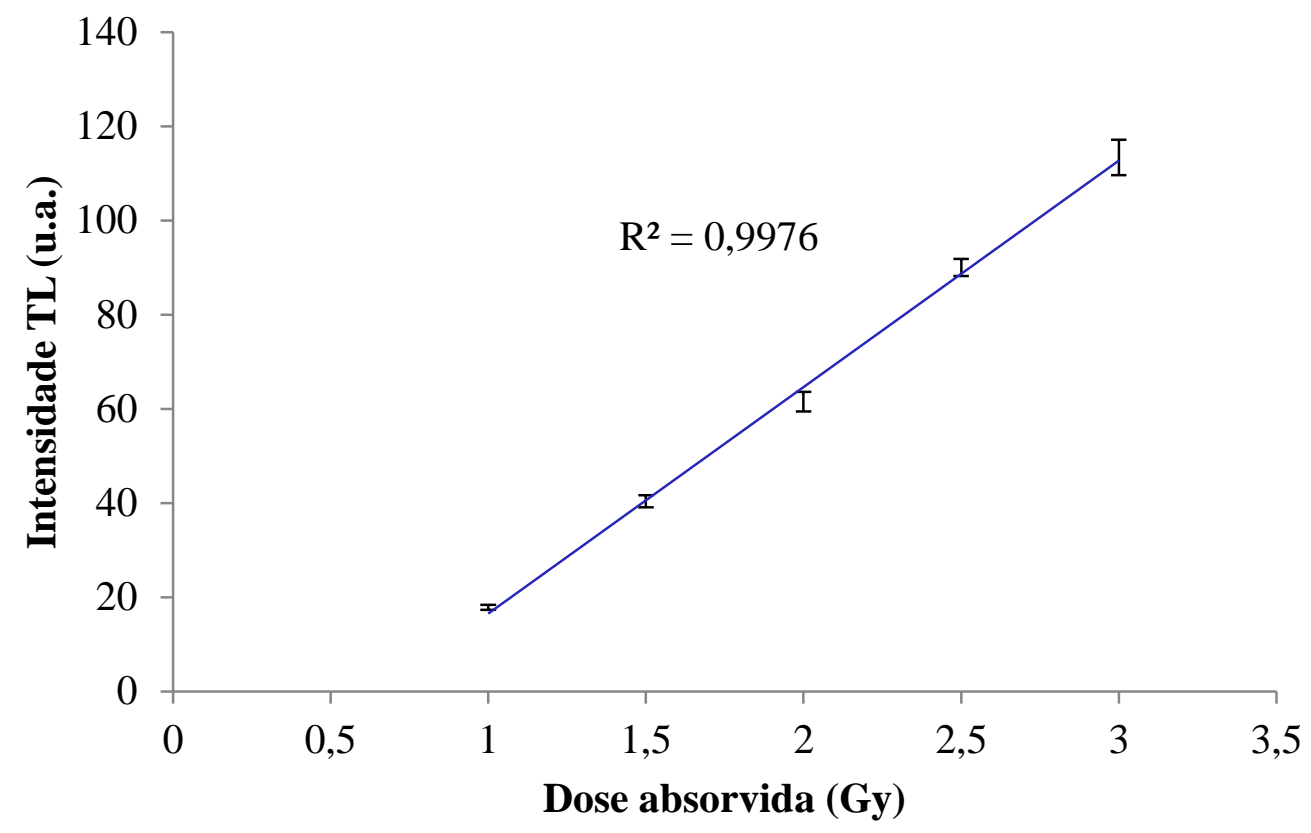

Figura 6: Curva dose-resposta das amostras de LiF-NRPB selecionadas.

Para se determinar o coeficiente de calibração $\left(\mathrm{F}_{\mathrm{c}}\right)$ para a intensidade TL das amostras de LiF-NRPB selecionadas, obtido pelo ajuste no método dos mínimos quadrados [2, 3], foi utilizada a Equação 3 e o valor correspondente está apresentado na Tabela 1, comparando-o ao coeficiente angular da curva dose-resposta da Figura 6.

$$
F_{c}=(\text { Dose }) /\left(\text { Leitura }_{\text {valor médio }}\right)
$$

Tabela 1: Coeficiente de calibração e coeficiente angular para as amostras de LiF-NRPB.

\begin{tabular}{c|c|c}
\hline $\begin{array}{c}\text { Coeficiente de } \\
\text { calibração } \mathbf{F}_{\mathbf{c}}\end{array}$ & $\begin{array}{c}\text { Coeficiente } \\
\text { angular }\end{array}$ & $\begin{array}{c}\text { Diferença } \\
\left(\mathbf{G y} / \mathbf{1 0}^{\mathbf{3}} \text { u.a }\right)\end{array}$ \\
\hline $0,0732 \pm 0,042$ & $0,0765 \pm 0,033$ & $(\boldsymbol{\%})$ \\
\hline
\end{tabular}


O coeficiente de calibração do LiF-NRPB para TL apresentou bom resultado, com uma diferença percentual máxima de 4,3\% quando comparado ao valor do coeficiente angular do ajuste linear da curva dose-resposta. O valor de $\mathrm{R}^{2}$ ficou em torno de 0,9976 , o que garantiu o ajuste linear da curva dose-resposta.

\subsection{Dependência angular}

Foi realizado o teste de dependência angular das amostras de LiF-NRPB com uma dose de 0,5 Gy, com uma repetição de 6 medições para cada angulação, por meio do sistema de radiação BSS1 e por meio de um goniômetro que permite a rotação da base que sustenta as amostras à frente da fonte radioativa.

A Tabela 2 apresenta os valores dos fatores de correção (Equação 4) para as diferentes angulações.

$$
\mathrm{F}_{\mathrm{c}}=\left(\mathrm{D}_{0}\right) /\left(\mathrm{D}_{\mathrm{ang}}\right)
$$

onde $\mathrm{D}_{0}$ é o valor da medição da luminescência com as amostras irradiadas a $0^{\circ}$ de inclinação e a $\mathrm{D}_{\text {ang }}$ é a medição da luminescência com uma angulação diferente de $0^{\circ}$.

Tabela 2: Fatores de correção para a dependência angular das 4 amostras de LiF-NRPB selecionadas.

\begin{tabular}{c|c|c|c}
\hline & \multicolumn{3}{|c}{ Fatores de correção para angulação } \\
\hline Amostra & $\mathbf{1 5}^{\circ}$ & $\mathbf{3 0}^{\circ}$ & $\mathbf{4 5}^{\circ}$ \\
\hline 1 & $1,03 \pm 0,07$ & $1,06 \pm 0,15$ & $1,11 \pm 0,15$ \\
\hline 2 & $1,01 \pm 0,04$ & $1,05 \pm 0,12$ & $1,10 \pm 0,13$ \\
\hline 3 & $1,03 \pm 0,03$ & $1,03 \pm 0,22$ & $1,11 \pm 0,20$ \\
\hline 4 & $1,02 \pm 0,05$ & $1,03 \pm 0,18$ & $1,12 \pm 0,17$ \\
\hline
\end{tabular}


Com o aumento da angulação, observou-se diminuição da resposta luminescente do material e esta diferença foi considerada nos cálculos das incertezas para aplicação do material na calibração do aplicador clínico.

\subsection{Calibração de um aplicador clínico}

O aplicador clínico dermatológico quadrado Amersham/SIQ18 foi calibrado por meio das amostras de melhor reprodutibilidade e homogeneidade de resposta com um valor médio obtido de 3 medições. Foi possível comparar os valores obtidos nas medições com o valor fornecido pelo fabricante $\left(0,027 \mathrm{Gys}^{-1}, 16 / 06 / 2019\right)$. A Tabela 3 apresenta o valor de taxa de dose obtido neste trabalho e a diferença entre o valor fornecido pelo fabricante.

Tabela 3: Calibração do aplicador Amersham/SIQ18 a 1 mm de distância. Valor médio de 3 medições.

\begin{tabular}{c|c|c}
\hline $\begin{array}{c}\text { Taxa de dose na } \\
\text { superfície da pele } \\
(\mathbf{G y} / \mathbf{s})\end{array}$ & $\begin{array}{c}\boldsymbol{\sigma}_{\text {expandida }} \\
\mathbf{k = 2}\end{array}$ & $\begin{array}{c}\Delta \\
\mathbf{( \% )}\end{array}$ \\
\hline 0,015 & 19,5 & 44,4 \\
\hline
\end{tabular}

$\Delta$ : Diferença entre os valores do certificado e o obtido no presente trabalho

O aplicador Amersham foi recalibrado por meio das amostras de LiF-NRPB e do sistema BSS2. O valor médio da taxa de dose obtido foi de 0,015 $\mathrm{Gys}^{-1}$, com uma incerteza de 19,5\%. Comparando com o valor da taxa de dose fornecida no certificado de calibração do fabricante, a diferença percentual foi de 44,4\%. Este valor alto pôde ser explicado a partir de 2003, pois ocorreram mudanças importantes no procedimento de calibração, quando comparada à maneira que os fabricantes calibravam estas fontes [10], e as mudanças são descritas pela norma ISO 21439:2009 [9]. As incertezas estão de acordo com o esperado pela norma, variando de 10\% a 20\%. Apesar da norma recomendar que o detector possua no máximo $1 \mathrm{~mm}$ de dimensões (para minimizar incertezas e aumentar a precisão das medições) o uso do LiF-NRPB foi dentro do esperado, principalmente pela pequena espessura que apresenta, menor que $1 \mathrm{~mm}$, e foi importante para se avaliar o material quanto às suas condições de uso e possíveis aplicações e estudos futuros. 


\section{CONCLUSÕES}

As pastilhas de LiF-NRPB apresentaram boa reprodutibilidade de resposta, boa sensibilidade; linearidade da curva dose-resposta, com coeficiente de correlação linear igual a 0,9976. As amostras mostraram a possibilidade de sua aplicação na recalibração do aplicador clínico de betaterapia, comparando com a calibração realizada no ano de 1968 pelo fabricante. Apesar das medições apresentarem uma alta diferença entre as calibrações e alta incerteza, os valores estão de acordo com as recomendações ISO e IAEA. Esta caracterização dosimétrica foi importante para classificar o material quanto à sua aplicação e poderá ser ainda reutilizado para outros fins dosimétricos.

\section{AGRADECIMENTOS}

Os autores agradecem às agências de fomento CNEN, CNPq (Projeto 301335/2016-8), CAPES (Projeto 554/18) e à Health Protection Agency pela doação das amostras.

\section{REFERÊNCIAS}

[1] DENNIS, J. A.; MARSHALL, T. O.; SHAW, K. B. The NRPB's new dosimeter and dose record keeping services. NDT International, v. 9, n. 6, p. 306-310, 1976.

[2] McKEEVER, S. W. S. Thermoluminescence in Solids. Cambridge: Cambridge University Press, 1985.

[3] FURETTA, C. Handbook of Thermoluminescence. Singapore: World Scientific Publishing Co. Pte. Ltd, 2010.

[4] GONZÁlEZ, P. R.; AZORÍN, J. Thermoluminescent response of LiF: Mg, Cu, P+ PTFE prepared in Mexico to $90 \mathrm{Sr} / 90 \mathrm{Y}$ beta particles. Radiation Physics and Chemistry, v. 61, n. 3-6, p. 425-427, 2001.

[5] AZORÍN, J.; TORIJANO, E.; AZORÍN, C. Type testing of a locally made LiF: Mg, Ti+ PTFE TLD for its use as a personal dosimeter. Applied Radiation and Isotopes, v. 141, p. 246-249, 2018. 
[6] RIVERA-MONTALVO, T. Radiation therapy dosimetry system. Applied Radiation and Isotopes, v. 83, p. 204-209, 2014.

[7] RIVERA-MONTALVO, T. Diagnostic radiology dosimetry: Status and trends. Applied Radiation and Isotopes, v. 117, p. 74-81, 2016.

[8] FRIEDELL, H.L.; THOMAS, C.I.; KROHMER, J. S. Beta-ray application to the eye: with the description of an applicator utilizing ${ }^{90} \mathrm{Sr}$ and its clinical use. American Journal Ophthalmology, v.33, n.4, p. 525-535, 1950.

[9] ISO, INTERNATIONAL ORGANIZATION FOR STANDARDIZATION. Clinical Dosimetry - Beta Radiation Sources for Brachytherapy. ISO, Geneva, 2009. (ISO 21439:2009).

[10] IAEA, INTERNATIONAL ATOMIC ENERGY AGENCY. Standards and Codes of Practice in Medical Radiation Dosimetry. 2003 (IAEA-CN-96/72). 